Artículos

\title{
Conocimientos y actitudes de enfermería sobre promoción de la salud: una mirada de región *
}

\author{
Nursing Knowledge and Attitudes About Health Promotion: A Regional View \\ Conhecimentos e atitudes dos enfermeiros sobre a promoção da saúde: um olhar da região
}

Nelly Johanna Loboa Rodríguez a

DOI: https://doi.org/10.11144/Javeriana.ie20-1.caep

Universidad de los Llanos, Colombia

johannaloboa@unillanos.edu.co

Patricia Elizabeth León Saavedra

Universidad de los Llanos, Colombia

Fecha de recepción: 15 Junio 2016

Fecha de aprobación: 12 Diciembre 2016

\section{Resumen:}

Introducción: la promoción de la salud se configura como un medio para conseguir la salud, concepto que involucra la articulación de conocimientos, actitudes, infraestructura, servicios, recursos y habilidades Objetivo: describir los conocimientos y actitudes sobre promoción de la salud que tienen las enfermeras en programas de atención integral al niño sano en instituciones prestadoras de servicios en el departamento del Meta, durante el 2013. Material y método: estudio cualitativo exploratorio-descriptivo con muestreo intencional opinático. Se realizó un análisis de contenido a partir de la información obtenida de entrevistas semiestructuradas grabadas, transcritas y validadas. Resultados: se trabajó con tres categorías de análisis: 1) impacto de la promoción de la salud, 2) herramientas para proporcionar cuidado de enfermería en la promoción de la salud y 3) dispositivos que inciden en el desarrollo de la promoción de la salud. Conclusiones: la promoción de la salud es un proceso encaminado a realizar, en conjunto, acciones destinadas a mejorar las condiciones y calidad de vida, implementando actitudes positivas y coherentes al quehacer de las enfermeras. Ello es de amplia importancia en la atención a la población infantil que aporta a la rentabilidad social del país.

Palabras clave: conocimientos, actitudes y prácticas en salud, enfermería, promoción de la salud.

\section{Abstract:}

Introduction: Health promotion is a way to achieve health, a concept that involves the articulation of knowledge, attitudes, infrastructure, services, resources and skills. Objective: To describe the knowledge and attitudes about health promotion that nurses have in comprehensive care programs for the healthy child in institutions providing services in the department of Meta, during the year 2013. Material and Method: Qualitative exploratory-descriptive study with intentional sampling. Content analysis was performed based on the information obtained from semi-structured interviews recorded, transcribed and validated. Results: Three categories of analysis: Impact of health promotion, tools to provide nursing care in health promotion and devices that affect the development of health promotion. Conclusions: The promotion of health is a process aimed at carrying out joint actions aimed at improving conditions and quality of life, implemented from positive and consistent attitudes to the task of nurses of great importance in the care of the child population contributes to the social profitability of the country.

Keywords: knowledge, attitudes and practices in health, nursing, health promotion.

\section{Resumo:}

Introdução: a promoção da saúde é configurado como um meio para a saúde, um conceito que envolve a articulação de conhecimentos, atitudes, infra-estrutura, serviços, recursos e habilidades. Objetivo: descrever o conhecimento e atitudes sobre a promoção da saúde com enfermeiras em programas de atendimento integral à criança sadia em instituições que prestam serviços no departamento de Meta, durante 2013. Materiais e Métodos: estudo qualitativo exploratório-descritivo, com amostragem intencional baseada em opinião. Análise de conteúdo foi realizada a partir das informações obtidas a partir de entrevistas semiestruturadas gravadas, transcritas e validados. Resultados: três categorias de análise: Impacto da promoção da saúde, ferramentas para prestar cuidados de enfermagem na promoção da saúde e dispositivos que afetam o desenvolvimento da promoção da saúde. Conclusões: a promoção da saúde é um processo de fazer em conjunto, acções que visam a melhoria das condições e qualidade de vida, implementado a partir de atitudes positivas e consistentes para o trabalho dos enfermeiros grande importância no cuidado da população infantil Ela contribui para a rentabilidade social do país.

Notas de autor 
Palavras-chave: conhecimento, atitudes e práticas em saúde, nutrição, promoção da saúde.

\section{Introducción}

A mediados del siglo XX, con Henry Sigerist, la promoción de la salud se ubicó como una de las tareas fundamentales de la medicina. Se planteó que la salud se promueve proporcionando condiciones de vida decentes, buenas condiciones de trabajo, educación, cultura física y los medios para el descanso y la recreación [1], y para este proceso es indispensable el trabajo conjunto de grupos políticos, sociales, económicos y, en especial, los trabajadores de la salud, quienes serían los encargados de definir las normas y de establecer los patrones para el mejoramiento de la salud.

A partir de la Carta de Ottawa [2], la promoción de la salud ingresa a un ciclo de afianzamiento teórico-conceptual y, al tiempo, se difunde como tendencia social, política e institucional; así se desarrollan escenarios, métodos y estrategias que se expresan en las conferencias internacionales y en diversas experiencias nacionales y locales [3]. Ello implica la elaboración de una política sana, la creación de ambientes favorables, el reforzamiento de la acción comunitaria, el desarrollo de actitudes personales y la reorientación de los servicios sanitarios.

La promoción de la salud puede desarrollarse desde la dimensión individual o poblacional. Al respecto, Restrepo [4] argumenta que hay algunas intervenciones promocionales en el ámbito personal dirigidas a lograr cambios conductuales que genéricamente se conocen como estilos de vida. El ámbito más social, más "estructural", es donde se plantean las acciones para modificar los ambientes físicos, sociales, culturales y políticos, para influir en las causas últimas o determinantes de las condiciones de vida/salud. Lo importante es que ambos niveles estén conectados, porque no se pueden concebir actividades educativas dirigidas al individuo para que cambie su comportamiento, separadas del marco de políticas públicas saludables y de procesos participativos de empoderamiento.

Enfermería, como disciplina social que encaminó su mirada a la comprensión de la salud y la enfermedad, confronta hoy la necesidad de redescubrir el nuevo aporte al cuidado y da cuenta de ello, mediante la comprensión de los aspectos relacionados con el proceso de la vida, la muerte, la sociedad y la cultura como hechos sociales de vital importancia en la transformación social. En este sentido, es parte de la contribución actual el análisis y la reflexión crítica sobre el sentido y el significado de cuidado de enfermería en el momento actual. Tales aspectos han avanzado con el desarrollo de teorías y modelos relacionados con la promoción de la salud, donde se destaca el modelo de Nola J. Pender, el cual planteó que promover un estado óptimo de salud era un objetivo que debía anteponerse a las acciones preventivas. Por ello, su planteamiento individual y colectivo favorece conductas saludables dentro de cada comunidad y, por ende, su concepción de salud parte de un componente altamente positivo, comprensivo y humanístico; toma a la persona como ser integral; y analiza los estilos de vida, las fortalezas y las capacidades de la gente en la toma de decisiones con respecto a su salud y su vida [1,5].

La enfermera y la persona se encuentran para llevar a cabo en conjunto una serie de acciones dirigidas a promover la salud y prevenir la enfermedad. Aspectos considerados en la Ley 266 de 1996 [6], cuando expone en su definición y propósitos que el ejercicio de la profesión de enfermería tiene como meta general promover la salud, entre otros.

Una nación debe resolver los problemas de la infancia si quiere ser exitosa en su lucha por alcanzar el verdadero desarrollo [7]. Es competencia de enfermería asumir el compromiso social que representa el control de crecimiento y desarrollo en los niños menores de diez años, que permita un abordaje eficaz en la prestación de los servicios, ofreciendo los recursos suficientes para la promoción y el fortalecimiento de los factores protectores, la prevención y el control de los factores de riesgo. 
Teniendo en cuenta lo anterior, el presente artículo describe los conocimientos y las actitudes sobre promoción de la salud que tienen las enfermeras que realizaron consulta de promoción de la salud y prevención de la enfermedad en programas de atención integral al niño sano en instituciones prestadoras de servicios (IPS) en la subregión Río Meta, en el departamento del Meta, durante el 2013.

\section{Materiales y métodos}

Este estudio tuvo un enfoque cualitativo de tipo exploratorio-descriptivo, por cuanto buscó describir los conocimientos y las actitudes sobre promoción de la salud de las enfermeras que realizaron consulta de promoción de la salud y prevención de la enfermedad en programas de atención integral al niño sano en IPS de la subregión Río Meta. El proceso de recolección, análisis y emisión de resultados se desarrolló entre febrero y diciembre del 2013 con la vinculación de dos estudiantes principiantes de investigación en opción de grado del Programa de Enfermería de la Facultad de Ciencias de la Salud de la Universidad de los Llanos.

La muestra se estableció de modo intencional opinático y estuvo determinada por el criterio estratégico personal de voluntariedad para participar en el estudio; de acuerdo con esto, los sujetos abordados fueron seis enfermeras que realizaron consulta de promoción de la salud y prevención de la enfermedad en el programa de atención integral al niño sano en IPS de la subregión del Río Meta, durante el 2013. El estudio fundamentó su desarrollo en las consideraciones éticas estipuladas para el desarrollo de procesos de investigación con seres humanos, según la Resolución 8430 de 1993 del Ministerio de Salud de Colombia [8], a partir de lo cual se cataloga como una investigación sin riesgo, que contó con aval del Comité de Ética de la Dirección General de Investigaciones de la Universidad de los Llanos.

Se solicitó el diligenciamiento por escrito del formato de consentimiento informado a cada uno de los sujetos de investigación, estando bajo anonimato, luego se procedió a la asignación de códigos a las entrevistas semiestructuradas con el fin de reservar la confidencialidad de los datos reportados. Las entrevistas se grabaron, transcribieron y luego las revisaron las participantes, para su validación.

Una vez recolectados los datos, se utilizó como proceso de codificación el análisis de contenido, de forma manual. Esta metodología de análisis cualitativo permite interpretar los datos recolectados en el transcurso de la investigación a partir de la búsqueda de los significados y sentidos que los sujetos de investigación perciben de su realidad [9].

El proceso de análisis de contenido fue realizado en dos momentos: sintáctico y semántico. En el primero se presentan los datos una vez segmentados como unidades de registros, de acuerdo con la categoría central, y en el segundo se agrupan los datos según pertinencia y relación entre los contenidos de las unidades [10].

\section{Resultados}

En la tabla 1 se presenta la síntesis de la categoría correspondiente al conocimiento y actitudes sobre promoción de la salud de las enfermeras abordadas. 
TABLA 1

Matriz categorial: conocimientos y actitudes sobre promoción de la salud que tienen las enfermeras. Subregión del Río Meta, 2013

\begin{tabular}{|c|c|c|c|}
\hline \multicolumn{4}{|c|}{$\begin{array}{c}\text { Categoría central } \\
\text { Conocimientos y actitudes sobre promoción de la salud que tienen las enfermeras }\end{array}$} \\
\hline Subcategorías & Núcleos temáticos & Fuente & Instrumento \\
\hline \multirow{3}{*}{$\begin{array}{l}\text { Impacto de la } \\
\text { promoción de la } \\
\text { salud }\end{array}$} & $\begin{array}{l}\text { Importancia social de la promoción de la } \\
\text { salud }\end{array}$ & \multirow{8}{*}{ 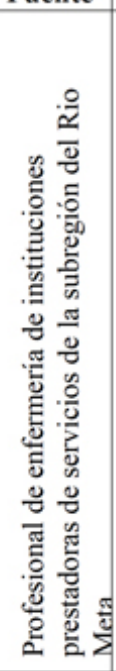 } & \multirow{8}{*}{ 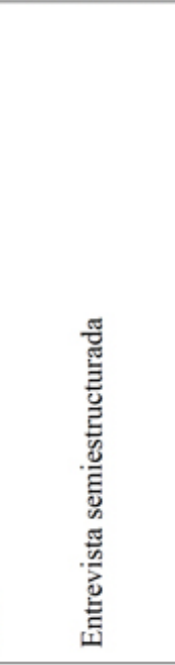 } \\
\hline & $\begin{array}{l}\text { Rentabilidad social sobre el mantenimiento } \\
\text { de la salud }\end{array}$ & & \\
\hline & $\begin{array}{l}\text { Consecuencias de la falta de acceso de los } \\
\text { niños y niñas a intervenciones de promoción } \\
\text { de la salud }\end{array}$ & & \\
\hline \multirow{2}{*}{$\begin{array}{l}\text { Acciones para } \\
\text { proporcionar } \\
\text { cuidado de } \\
\text { enfermería en la } \\
\text { promoción de la } \\
\text { salud }\end{array}$} & $\begin{array}{l}\text { Detección de factores de riesgo y factores de } \\
\text { protección en niños y niñas }\end{array}$ & & \\
\hline & $\begin{array}{l}\text { Empatía y la relación de ayuda en el cuidado } \\
\text { de enfermería en promoción de la salud }\end{array}$ & & \\
\hline \multirow{3}{*}{$\begin{array}{l}\text { Dispositivos } \\
\text { que inciden en } \\
\text { el desarrollo de } \\
\text { la promoción de } \\
\text { la salud }\end{array}$} & $\begin{array}{l}\text { Educación esencial en el actuar en } \\
\text { promoción de la salud }\end{array}$ & & \\
\hline & $\begin{array}{l}\text { Las tensiones del desarrollo de la promoción } \\
\text { de la salud en el marco de las politicas }\end{array}$ & & \\
\hline & $\begin{array}{l}\text { Trabajo intersectorial que facilita el } \\
\text { desarrollo de la promoción de la salud }\end{array}$ & & \\
\hline
\end{tabular}

Fuente: elaboración propia.

El primer núcleo temático de la subcategoría denominada impacto de la promoción de la salud agrupa los datos relacionados con el primer núcleo temático, que es la importancia social de la promoción de la salud, en el que se identificaron expresiones como: "la base principal de la atención integral del niño sano es la promoción de la salud" (E2C1P1); "sí, yo sé, y reconozco la importancia de ese programa, sé qué es lo que me permite" (E4C77P7); "si la promoción de salud se hace de forma adecuada y logra su objetivo de prevenir enfermedades, influye en que los niños crezcan sanos" (E2C4P1); "si tenemos niños aliviados, no vamos a tener tantos costos en tratamientos, ni hospitalizaciones, ni vamos a disminuir su productividad de su propia familia" (E4C14P1); "la promoción de la salud es supremamente importante, y más ahora que ha tomado tanta fuerza con la atención primaria en salud" (E6C1P1); "18 prácticas claves que promociona salud infantil" (E6C28P3); "es decir, que son programas de mínimo costo para la salud, para el Estado pero de un amplio beneficio social y en la salud, son muy rentables; pero si los ejecutamos de la mejor manera" (E6C9P1); "genera un impacto social positivo dentro de la gente" (E4C7P1); "por lo tanto vamos a tener unos jóvenes en el futuro, adolescentes y jóvenes productivos" (E4C12P1); "amplio beneficio social a un mínimo costo" (E6C7P1); "a pesar de que pueden generar algún costo para la salud, pero que alguna u otra manera genere un beneficio también para la sociedad y para la población" (E6C5P1).

El segundo núcleo agrupa los datos relacionados con la rentabilidad social sobre el mantenimiento de la salud en los niños y niñas atendidos integralmente en el programa de crecimiento y desarrollo, asociado al aprovechamiento de los recursos al ejecutar las actividades de promoción de salud en la población: "rentabilidad social lo obtenemos en los niños, pues son niños que los vamos a tener sanitos" (E5C10P1); "la manera en que yo puedo hacer más, gastando los mismos recursos" (E3C5P1); "genera ingresos que le permiten mantenerse, seguir prestando el servicio en forma indefinida y es auto sostenible" (E4C8P1); "a disminuir costos a nivel de la prestación de los servicios y asistenciales" (E4C13P1); "hay que enfatizar en el empoderamiento de la salud y en el autocuidado" (E5C44P4); "siempre buscamos que las personas se apropien del cuidado de su salud" (E3C22P2); "queremos que en vez de que se gasten el dinero en la parte de medicamentos, más bien estén comprando que las frutas, que las verduras, las cosas que requiere el niño para mantenerse como niño sano" (E1C67P6). 
El tercer núcleo temático refleja la consideración de las enfermeras, relacionada con las consecuencias de la falta de acceso de los niños a intervenciones de promoción de la salud, lo que evidencia el impacto de la promoción de la salud, al reconocer las circunstancias que se presentan cuando no se desarrolla. Esto se manifiesta en expresiones como: "van a tener que gastar más plata o no van a tener tiempo; o van a faltar a su trabajo" (E2C6P1); "se va a enfermar con más frecuencia" (E6C37P4); "una mamá que no va a los controles con su niño, no va a aprender a identificar el riesgo de su niño y no va aprender hábitos saludables, razón por la cual el niño se va a ver afectado en su desarrollo" (E4C46P4); "no va a tener una mejor calidad de vida" (E4C35P3); "un niño que vamos a ver que se nos va a perder o que nos va a llegar a los dos o tres meses simplemente con las vacunas de recién nacido" (E6C34P3).

Con relación a la segunda subcategoría, denominada herramientas para proporcionar cuidado de enfermería en la promoción de la salud, el primer núcleo temático agrupa los datos que hacen referencia a la detección de factores de riesgo y factores de protección en niños y niñas, que se describe a partir de la descripción de acciones que desarrolla el profesional de enfermería y que puede incidir en evitar complicaciones y mantener la salud de los niños, manifestados a través de las siguientes expresiones: "si en el momento de la consulta miro que el niño posee alguna alteración en su salud, oriento a la madre y lo remito" (E1C51P5); "actuar sobre ellos y evitar que se conviertan en algo irreparable" (E2C3P1); "para los papitos es mejor llevarlos a una consulta de control y no mantener con ellos enfermos en un hospital o en urgencias" (E2C5P1); "las actividades que desarrollamos en la consulta están encaminadas a detectar los riesgos o los problemas de manera temprana" (E5C5P3); "permite identificar todos aquellos factores protectores" (E6C5P2); "identificamos los factores de riesgo y factores protectores que identificamos dentro la consulta o dentro de su familia y su comunidad" (E1C31P3); "mis actividades están encaminadas a cómo prevenir los factores de riesgo, e identificar también los factores protectores de la familia" (E2C19P2); "identifico que los niños que me llegan, o las mayores causas de consulta que me llega al centro de salud son por EDA, en ciertos periodos de tiempo, yo tengo primero que investigar qué es lo que está pasando en esa zona, porque es que están presentando sus casos" (E3C40P4).

El segundo núcleo temático agrupa los datos relacionados con la empatía y la relación de ayuda en el cuidado de enfermería en promoción de la salud, evidenciado en expresiones como: "ofrecerles toda nuestra parte técnica que nos permite ayudar en la promoción de la salud" (E3C10P2); "cómo yo ayudo a esa mamá, es mi principal interrogante" (E3C40P4); "una relación más de un vínculo que lo lleve a ayudar" (E6C40P4); “bastante porque se gana la empatía del menor y la empatía de la mamá” (E33C11P1); "el estado de ánimo sí influye, pero yo no puedo estar de mal genio; esos sentimientos se quedan en el profesional" (E4C15P2); "por más malgeniada, por más estresado que estén en la casa, lo que sea aquí en el trabajo es otra historia" (E4C20P2); "allá en la consulta no importa uno, sino importa el niño y su cuidador" (E4C18P2); "específicamente importante porque, cuando uno habla con las personas; uno debe hablarles de la forma que mejor entiendan" (E6C3P1); "se apoyan mucho en uno y uno tiene que brindar mucha confianza" (E5C4P1); "si uno tiene una buena relación, eso les genera confianza y para ellos va a ser mucho más fácil venir, dialogar con uno" (E5C6P1); "siempre para los pacientes usted tiene que tener una buena cara" (E1C67P6); "no es que sean difíciles, sino que como uno tiene que ser muy integral en la atención" (E3C10P2); "tengo que brindarle o producirle esa empatía para que me permita tener confianza y consultarme todas sus necesidades y problemas frente al niño" (E6C14P2).

En la tercera subcategoría relacionada con dispositivos que inciden en el desarrollo de la promoción de la salud, el primer núcleo temático agrupa los datos de los profesionales de enfermería que hacen referencia a la educación para la salud desde su importancia para el desarrollo de la promoción de la salud. Las expresiones, que relacionan esto son: "nosotros sí hacemos actividades educativas, charlas a los chicos sobre todo con los grupos que manejan mamás, que manejan lactantes, que tienen niños menores de 5 años, eso siempre lo hacemos" (E4C53P5); "la educación que uno les puede brindar debe ser clara y concisa" (E4C54P5); "todo proceso que uno intente realizar educativo, el objetivo general es generar un cambio de conducta del 
individuo" (E1C67P6); "nos permiten brindarle una educación al paciente, a la madre, a los cuidadores de los niños" (E3C49P4); "educar a la comunidad para que las madres, los padres y los cuidadores den atención de calidad en el hogar, porque es que en el hogar también se necesita un ambiente saludable” (E2C51P4); "necesitamos es educar y educación no se involucran recursos tecnológicos, ni recursos en medicamentos, sino básicamente dar una buena educación" (E1C54P5); "se debe dar en todos los espacios que tengamos con padres, madres y cuidadores y no perder ningún espacio" (E1C5OP5).

El segundo núcleo temático agrupa los datos relacionados con las tensiones del desarrollo de la promoción de la salud en el marco de las políticas públicas. Como aspecto negativo, al evidenciar que no debería existir limitación en el desarrollo de las actividades propuestas por el programa de atención al niño sano, las expresiones que fundamentan esto son: "hasta el momento hay muchas barreras" (E5C21P2); "no debería haber ninguna barrera como lo es: si no tiene la EPS, que si no le dieron la autorización" (E4C2P1); "evitar que hayan tantas barreras para acceder a los servicios" (E3C10P1); "esa parte se trabaja mucho con la comunidad para evitar que se congestionen así los servicios de salud" (E2C47P4); "a veces es demorado el inicio de la consulta, entonces ellos ya vienen de mal genio" (E1C41P4); "la salud no depende del sistema y no depende de nosotros como funcionarios" (E5C25P3); "es una de las estrategias más importantes dentro de las políticas nacionales" (E6C29P3); "todos los niños y cuidadores son diferentes" (E5C7P1).

El tercer núcleo temático agrupa frases relacionadas con el trabajo intersectorial, que facilita el desarrollo de la promoción de la salud en el marco de la salud pública, a partir de la articulación de acciones que propenden a la búsqueda del mantenimiento de la salud de los niños y niñas desde diferentes escenarios y actores. Ello se refleja en las siguientes manifestaciones: "hay una red amplia como el FAMI y las familias en acción" (E4C32P5); "con los colegios como tal de se trata de desarrollar actividades de educación con los niños" (E1C67P6); "cuando los remitimos a salud oral, a vacunación" (E2C3P1); "se refiere al trabajo interdisciplinario que podemos hacer nosotros, de ir a los colegios, de hacer actividades; o articularnos con los profesores" (E3C4P1); "sistema educativo y el sistema de salud van de la mano porque uno hace promoción de la salud a través de la educación" (E1C40P4); "lo ideal es involucrar a otros actores sociales que los presidentes de acción comunal, las madres líderes, las madres del ICBF, la policía comunitaria, la iglesia, educación, todos esos actores de alguna u otra manera se involucran" (E1C67P6); "se debe hacer involucrando diferentes actores sociales" (E4C47P4); "la responsabilidad de hacer promoción de la salud, no es solamente del sector salud, la responsabilidad es de todos" (E5C21P2).

\section{Discusión}

En Colombia, Velásquez y colegas [11] sustentan que la promoción de la salud es una de las estrategias que desarrolla y fortalece la ciudadanía, de modo que la población pueda ejercer sus derechos en salud. Reconocieron que la participación de la comunidad es un elemento fundamental para fomentar el autocuidado de la salud y para establecer una relación más horizontal entre los servicios de salud y la comunidad.

Las enfermeras de la subregión del Río Meta consideran que sus conocimientos y actitudes sobre promoción de la salud se encuentran enmarcadas en tres subcategorías de análisis, las cuales se describen a continuación:

Las enfermeras definen el impacto de la promoción de la salud como aquellos resultados obtenidos en los campos físico, social y económico, luego de que los profesionales de la salud ejecuten acciones de promoción de salud, que involucran a la población en general. Dentro de esta subcategoría se identificaron tres núcleos temáticos agrupados. De forma similar, Maza, Zabala y Escobar [12] destacan la importancia de la presencia fuerte de la promoción de la salud, en el desarrollo de la población, cuando describe que el gran desafío es lograr que la promoción de la salud adquiera un carácter de política de Estado, que se exprese en 
políticas públicas con adecuadas regulaciones y mecanismos que permitan establecer con vigor procesos de participación ciudadana para el mejoramiento de la salud y calidad de vida.

Adicionalmente, el cumplimiento adecuado de las actividades, según las enfermeras abordadas, es lograr el objetivo de detectar de forma temprana los factores de riesgo presentes, que lleven a evitar cualquier alteración en la salud de los niños y, por tanto, a fortalecer la prevención de enfermedades prevalentes de la infancia y las situaciones adversas. Ello se evidencia a partir de las ventajas que implica el mantenimiento de la salud de los niños, para su familia y la sociedad, en los planos físico, económico y social. Al respecto, Díaz y colaboradores [13] refieren que la promoción de la salud en la sociedad es la estrategia encaminada a desarrollar procesos y actividades individuales o grupales con el fin de modificar conductas de riesgo y adoptar estilos de vida saludables.

Los resultados evidencian la rentabilidad social frente al mantenimiento de la salud en los niños que son atendidos integralmente en el programa de promoción de la salud, asociado a aprovechar los recursos en el desarrollo de las actividades de promoción de salud en la población. Ello se refleja en la aplicación de las acciones de promoción de la salud declaradas en la Declaración de Yakarta [14], cuando define que el rol del mantenimiento de la salud en el proceso de desarrollo de la sociedad está fundamentado en que la salud es un derecho humano básico e indispensable para el desarrollo social y económico, lo cual se evidencia en el desarrollo del programa de atención al niño sano, porque impacta en la población infantil, y ello beneficia al país, social y económicamente.

Por otra parte, la enfermera involucra la estrategia de Atención Primaria en Salud para la identificación de factores de riesgo y continuar con la intervención oportuna al niño sano, a fin de evitarle complicaciones. Ello coincide con lo expuesto por Camargo y Pinzón [15], cuando definen al profesional de enfermería como una persona capacitada para llevar a cabo una multitud de complejas funciones, utilizando de forma eficaz e independiente su capacidad de juicio para desempeñar sus responsabilidades.

La observación se reconoce como una herramienta importante para detectar factores de riesgo y protectores en el niño - acción importante del profesional, que incide en evitar complicaciones y mantener la salud del menor-. Fortuna y colegas [16] verificaron cómo muchas iniciativas se centran en la intervención sobre un factor de riesgo tomado de manera aislada, abordado de forma persuasiva y limitado al comportamiento; los autores concordaron en afirmar la relevancia del interés que se le presta a la determinación oportuna de los factores de riesgo y protectores, para generar acciones que propendan a la búsqueda o el fortalecimiento de conductas que beneficien la salud del niño sujeto de atención.

La empatía y la relación de ayuda en el cuidado de enfermería en promoción de la salud son importantes en el ejercicio del desempeño profesional. Tanto Roy y Andrews [17] como Pedraza [18] consideran que el ser de enfermería se enfoca hacia el autoconcepto que el profesional tiene de su profesión. Este se refiere el arte de cuidar desde el rol orientado al bienestar y satisfacción de las necesidades de la sociedad; prima aquí la orientación colectiva por encima de la orientación individual, pues, por encima de todo, para la mayoría su profesión consiste principalmente en una forma de ayudar a los demás.

A través de este estudio se ha destacado el enfoque de cuidado, aspecto que, según De Souza [19], refleja el atributo al ser: la empatía, la calidad humana, el compromiso y la responsabilidad hacia el individuo y la comunidad. De igual manera, el estudio de Latiera y colaboradores [20] considera que la relación de ayuda profesional de enfermería está sustentada en el cuidado de la salud, el cual es un proceso interactivo entre una persona que tiene necesidad de ayuda y otra capaz de ofrecérsela.

La educación, desde la importancia para el desarrollo de la promoción de la salud, se relaciona con lo descrito por Laguado [21] con relación a que los procesos de educación permiten fortalecer a los padres en los cuidados básicos con los hijos, en cuanto a estimulación, alimentación, higiene, hábitos saludables, medidas preventivas para evitar riesgos y, así mismo, contribuir al buen crecimiento y desarrollo de los niños. Carmona y Pichardo [22] expresan que el proceso de educación a la comunidad, para que sea eficaz, requiere mensajes claros, continuos, accesibles, con significado y recursos suficientes. 
Se identificaron tensiones del desarrollo de la promoción de la salud en el marco de las políticas públicas, relacionadas con las expresiones de las enfermeras respecto a que no debería existir limitación en el desarrollo de las actividades propuestas por el programa de atención al niño sano, en términos de recursos económicos y de tiempo para su desarrollo. Los anteriores aspectos evidencian que el impacto de la promoción de la salud depende de la capacidad de la enfermera para brindarla y de la población para aceptarla. Así lo exponen Ramos y colaboradores [23] en su estudio, cuando refieren que las relaciones de la enfermera en la sociedad se basan en un estado de equilibrio entre las habilidades de enfermería para prescribir, asignar, manejar y mantener los sistemas de autocuidado terapéutico de las personas y las capacidades de estas y de sus familiares para realizarlas; no obstante, los profesionales ponen en evidencia otros factores que también influyen en el desarrollo de este proceso, como los servicios de salud que, en gran medida, se ven afectados por la gran demanda de usuarios con presencia de alteraciones de salud, o la disminución de la asignación de recursos para el desarrollo de actividades dentro del programa de promoción de salud; entre otros.

Se identificó que el trabajo intersectorial facilita el desarrollo de la promoción de la salud en el marco de la salud pública, dada la articulación de las acciones en la búsqueda del mantenimiento de la salud de los niños y niñas desde diferentes escenarios. Dichos resultados muestran que la enfermera considera de gran importancia trabajar conjuntamente con otros sectores sociales, en la búsqueda del bienestar comunitario, uniendo esfuerzos para lograr el objetivo común. Este hallazgo tiene relación con los resultados del estudio de Roth y Molina [24], en el que se reconoce la importancia de la articulación y cooperación intersectorial e interinstitucional para mejorar los logros en la salud pública.

\section{Conclusiones}

Se evidenció que el impacto de la promoción de la salud se relaciona favorablemente con su importancia social y la rentabilidad social sobre el mantenimiento de la salud de los niños y niñas. En paralelo, se identificó que la falta de acceso de los niños a intervenciones de promoción de la salud es un aspecto que disminuye sustancialmente el desarrollo y crecimiento saludable de niños, niñas y familias.

Se identificó que entre las acciones que las enfermeras proporcionan para brindar cuidado en el marco de la promoción de la salud consideran que está la detección de factores de riesgo y factores de protección en los niños y niñas; así mismo, consideran acciones relevantes la generación de empatía y la relación de ayuda.

Finalmente, se identificó que hay dispositivos que inciden en el desarrollo de la promoción de la salud. De manera positiva se relaciona la educación para la salud y el trabajo intersectorial; en contraste, de manera negativa se relacionaron las tensiones del desarrollo de la promoción de la salud en el marco de la política, aspectos que favorecen o limitan el desempeño profesional, los cuales deben ser abordados desde diferentes instancias para lograr minimizarlos.

Conflicto de intereses

Las autoras declaran no tener ningún conflicto de intereses.

\section{Agradecimientos}

Esta investigación, contó con el apoyo financiero de la Dirección General de Investigaciones de la Universidad de los Llanos. 


\section{Referencias}

1. Aristizábal GP, Blanco DM, Sánchez A, Ostiguín RM. El modelo de promoción de la salud de Nola Pender: una reflexión en torno a su comprensión. Rev Enfem Universit [internet]. 2011;8(4):16-23. Disponible en: http:// www.revistas.unam.mx/index.php/reu/article/view/32991/30231

2. Organización Mundial de la Salud. Carta de Ottawa. En: Carta de Ottawa para la Promoción de la Salud [internet]. Ginebra: OMS; 1986. Disponible en: http://www.paho.org/spanish/HPP/OttawaCharterSp.pdf

3. Robledo MR, Agudelo C. Aproximación a la construcción teórica de la promoción de la salud. Rev Salud Pública [internet]. 2011;13(6):1031-50. Disponible en: http://www.scielo.org.co/scielo.php?script=sci_arttext\&pid= S0124-00642011000600015\&lng=es

4. Restrepo H. Promoción de la salud: como construir vida saludable. Bogotá: Editorial Médica Panamericana; 2001.

5. Giraldo O, Toro R, Macías L, Valencia G, Palacio R. La promoción de la salud como estrategia para el fomento de estilos de vida saludables. Hacia Promoc Salud [internet]. 2010;15(1):128-43. Disponible en: http://www.sciel o.org.co/scielo.php?script=sci_arttext\&pid=S0121-75772010000100010\&lng=es

6. Ley 266 de 1996, por la cual se reglamenta la profesión de Enfermería en Colombia y se dictan otras disposiciones. Congreso de la República de Colombia

7. Ministerio de la Protección Social de Colombia. Norma técnica para la detección temprana de las alteraciones del crecimiento y desarrollo en el menor de 10 años. 2000.

8. Resolución 008430 de 1993, de normas científicas, técnicas y administrativas para la investigación en salud. Ministerio de Salud de Colombia; 1993.

9. Strauss S, Corbin J. Bases de la investigación cualitativa: técnicas y procedimientos para desarrollar la teoría fundamentada. Medellín: Universidad de Antioquia; 2002.

10. Bautista NP. Proceso de la investigación cualitativa: epistemología, metodología y aplicaciones. Bogotá: Manual Moderno; 2011.

11. Velásquez VF, López AL, Puerto AH, Cataño N, Muñoz AI. Por un futuro mejor: programa de promoción de la salud para afrodescendientes. Rev Cuid [internet]. 2016;7(1):1185-94. Disponible en: http://dx.doi.org/10.15 649/cuidarte.v7i1.239

12. Maza M, Zavala M, Escobar JM. Actitud de enfermera ante la muerte de pacientes. Cienc Enferm [internet]. 2009;15(1):39-48. Disponible en: http://www.scielo.cl/scielo.php?pid=S071795532009000100006\&script= sci_arttext

13. Díaz Y, Pérez JL, Báez F, Conde M. Generalidades sobre promoción y educación para la salud. Rev Cubana Med Gen Integr [internet]. 2012;28(3):299-308. Disponible en: http://scielo.sld.cu/scielo.php?script=sci_arttext\& pid $=$ S0864-21252012000300009\&lng=es

14. Organización Mundial de la Salud. Declaración de Yakarta sobre la Promoción de la Salud en el Siglo XXI. Yakarta, República de Indonesia; 1997.

15. Camargo CM, Pinzón GY. La promoción de la salud en la primera infancia: evolución del concepto y su aplicación en el contexto internacional y nacional. Rev Fac Med [internet]. 2012;60(1):62-74. Disponible en: http://ww w.scielo.org.co/pdf/rfmun/v60s1/v60s1a07.pdf

16. Fortuna CM, Matumoto S, Pereira MJ, Mishima SM, Kawata LS, Camargo-Borges C. El enfermero y las prácticas de cuidados colectivos en la estrategia: salud de la familia. Rev Latino-am Enfermagem [internet]. 2011;19(3):1-8. Disponible en: http://www.scielo.br/pdf/rlae/v19n3/es_18.pdf

17. Roy S, Andrews H. The Roy adaptation model. Connecticut: Appleton \& Lange; 1999.

18. Pedraza R. Percepción de las enfermeras de Barcelona sobre la aplicación del rol autónomo en su contexto laboral. Cataluña: Universidad internacional de Cataluña; 2011.

19. De Souza MC. El desafío del conocimiento: investigación cualitativa en salud. Buenos Aires: Lugar; 1997.

20. Latiera P, García R, Casas F, Díaz C, Gómez JI, Salvadores P. Grado de conocimientos y actitudes de la enfermería de la comunidad Autónoma de Madrid ante el dolor: diplomados en enfermería y profesionales. Rev Soc Esp 
Dolor [internet]. 2012;6(1):293-300. Disponible en: http://scielo.isciii.es/scielo.php?script=sci_arttext\&pid= S1134-80462012000600003

21. Laguado E. Cuidado de enfermería a padres para fortalecer el crecimiento y desarrollo de sus hijos. Rev Cuid [internet]. 2013;4(1):550-7. Disponible en: http://www.revistacuidarte.org/index.php/cuidarte/article/view/ $17 / 146$

22. Carmona M, Pichardo M. Actitudes de enfermera hacia el comportamiento suicida: Influencia de la inteligencia emocional. Rev Latino-Am Enfermagem [internet]. 2012;20(6):1161-9. Disponible en: http://www.scielo.br/ pdf/rlae/v20n6/es_19.pdf

23. Ramos A, Ruzafa M, Fernández S, Del Pino R, Armero D. Actitudes de médicos y enfermeras ante las actividades preventivas y de promoción en atención primaria. Aten. Primaria. 2014;46(9):483-91.

24. Roth AN, Molina G. Rectoría y gobernanza en salud pública en el contexto del sistema de salud colombiano, 2012-2013. Rev Salud Pública. 2013;15(1):44-55.

\section{Notas}

* Artículo original de investigación.

\section{BY}

\section{Información adicional}

Cómo citar: Loboa Rodríguez NJ, León Saavedra PE. Conocimientos y actitudes de enfermería sobre promoción de la salud: una mirada de región. Investig Enferm Imagen Desarr. 2018;20(1):xx-xx. https://d oi.org/10.11144/Javeriana.ie20-1.caep 